\title{
Totally laparoscopic feeding jejunostomy - a technique modification
}

\author{
Maciej Bobowicz, Wojciech Makarewicz, Tomasz Polec, Arkadiusz Kopiejć, Tomasz Jastrzębski, Jacek Zieliński, \\ Janusz Jaśkiewicz
}

Department of Oncological Surgery, Hospital of Medical University of Gdansk, Poland

Videosurgery and Other Miniinvasive Techniques 2011; 6 (4): 256-260 DOI: 10.5114/wiitm.2011.26262

\begin{abstract}
In oncological patients with upper gastrointestinal tract tumours, dysphagia and cachexy necessitate gastrostomy or jejunostomy as the only options of enteral access for long-term feeding. In this article the authors describe a modified technique of laparoscopic feeding jejunostomy applied during the staging laparoscopy. A 48-year-old male patient with gastroesophageal junction tumour and a 68-year-old male patient with oesophageal tumour were operated on using the described technique. Exploratory laparoscopy was performed. Then the feeding jejunostomy was made using a Cystofix ${ }^{\circledR}$ TUR catheter. The jejunum was fixed to the abdominal wall with four 2.0 Novafil ${ }^{T M}$ transabdominal stitches. Two additional sutures were placed caudally about $4 \mathrm{~cm}$ and $8 \mathrm{~cm}$ from the jejunostomy, aiming at prevention of jejunal torsion. Total operating time was $45 \mathrm{~min}$. There was no blood loss. There were no intraoperative complications. The only adverse event was one jejunostomy wound infection that responded well to oral antibiotics. There were no mortalities. The described technique has most of the benefits of laparoscopic feeding jejunostomy with some steps added from the open operation making the procedure easier to perform as part of a staging operation with a relatively short additional operating time. The proposed transabdominal stitches make the technique easier to apply. Two additional 'anti-torsion sutures' prevent postoperative volvulus. Use of the Cystofix catheter allows easy introduction of the catheter into the peritoneal cavity and the jejunal lumen, providing a good seal at the same time. Further studies on larger groups of patients are required to assess long-term outcomes of the proposed modified technique.
\end{abstract}

Key words: feeding microjejunostomy, enteral feeding, laparoscopy, minimally invasive surgery, technique modification, oncology.

\section{Introduction}

Many oncological patients with upper gastrointestinal (GI) tract tumours, apart from other symptoms, are malnourished or cachectic at the time of presentation. In these patients feeding plays a crucial role, including as part of palliative treatment. Many studies have proved the benefits of enteral feeding over parenteral if feasible [1-3]. Depending on the tumour's location and clinical stage there are several options of enteral feed- ing aids available. Since the introduction of percutaneous endoscopic gastrostomy (PEG) and its relatively easy application in most patients, older techniques such as open gastrostomy or jejunostomy have rather few indications. The majority of non-PEG techniques are used in patients with upper digestive tract, head and neck tumours or trauma that renders the PEG technique unfeasible or unsafe for the patient [4].

In these patients, especially with advanced disease requiring neoadjuvant chemotherapy or pallia- 
tive treatment, open gastrostomy and jejunostomy were the only options of enteral access. Since the first report of laparoscopic jejunostomy by O'Regan et al. in 1990 there have been several publications presenting techniques and outcomes of laparoscopic feeding jejunostomy [5]. Laparoscopic jejunostomy can accompany staging or diagnostic laparoscopy for upper GI malignancy when the disease appears advanced, hence avoiding additional anaesthesia and an operation in the near future.

In this article the authors describe the technique of laparoscopic feeding jejunostomy applied during the staging laparoscopy in two consecutive patients with advanced upper gastrointestinal tract cancer with co-morbid cachexy, requiring enteral feeding and neoadjuvant chemotherapy.

\section{Case reports}

\section{Case 1}

A 48-year-old male patient was referred due to cachexy with weight loss of almost $22 \mathrm{~kg}$ in 2 months. At presentation the patient was unable to eat either solid or liquid food and was only able to drink clear water. There were no other co-morbid conditions. The patient had a subsequent gastroscopy and a computed tomography scan (CT). Gastroscopy showed gastroesophageal junction tumour almost completely obstructing the gastric inlet. Samples of the tumour mass were taken but the pathology result was unavailable at the time of surgery. Computed tomography scan showed thickening of the gastric wall up to $12 \mathrm{~mm}$ with local lymphadenopathy (largest lymph nodes up to $19 \mathrm{~mm}$ ).

\section{Case 2}

A 68-year-old male patient was admitted due to cachexy with weight loss of $17 \mathrm{~kg}$ in 6 months. At presentation the patient was unable to eat or drink. The patient had well-controlled arterial hypertension. He had subsequent gastroscopy and a CT. Gastroscopy showed a large oesophageal tumour (involving the lower $6 \mathrm{~cm}$ of the oesophagus) irregularly constricting the gastric cardia. Pathology examination of samples showed squamous cell carcinoma G1 of the oesophagus. Computed tomography scan showed thickening of the oesophageal wall up to $30 \mathrm{~mm}$ with mediastinal lymphadenopathy (largest lymph nodes up to $18 \mathrm{~mm}$ ).
Due to the advanced stage of the tumour, dysphagia and severe cachexy in both patients, the decision on staging laparoscopy and feeding laparoscopic microjejunostomy was made. Informed consent was obtained according to the local standards.

\section{Preoperative period}

Standard preoperative antibiotic prophylaxis with $2 \mathrm{~g}$ of cefuroxime i.v. and $0.5 \mathrm{~g}$ of metronidazole was given $30 \mathrm{~min}$ prior to the operation. Antithrombotic prophylaxis including mechanical and pharmacological (5000 i.u. of dalteparin sodium) prophylaxis was applied.

\section{Patient's positioning}

The procedure was performed under general anaesthesia. The patient was placed in the prone position with arms spread to the sides. The operator together with the assistant was standing on the right side of the patient whereas the scrub nurse was on the left side of the patient. The laparoscopy set was placed on the left side of the patient.

\section{Trocar placement}

One $10 \mathrm{~mm}$ trocar and two $5 \mathrm{~mm}$ trocars were used. The $10 \mathrm{~mm}$ trocar was placed using an open Hassan technique in the intersection of the line of the umbilicus and the right sterno-clavicular line. Both $5 \mathrm{~mm}$ trocars were placed under direct vision in the right sterno-clavicular line about $7 \mathrm{~cm}$ above and below the horizontal line crossing the umbilicus (Figure $1 \mathrm{~A}$ ).

Later during the procedure 15 Ch Cystofix ${ }^{\circledR}$ TUR (B. Braun Melsungen AG, Melsungen, Germany) was inserted with the integral introducer at the point of the intersection of the left mid-clavicular line with a vertical line about $2 \mathrm{~cm}$ below the umbilicus (Figure 1).

\section{Staging laparoscopy}

$\mathrm{CO}_{2}$ pneumoperitoneum of $12 \mathrm{mmHg}$ was achieved. Exploratory laparoscopy with thorough abdominal cavity inspection was performed using the $30^{\circ}$ endoscope camera and standard laparoscopic instruments to assess the tumour size and its spread as well as possible metastases (Figure 2). Peritoneal fluid was aspirated for pathology examination (tumour cells) as well as for Ca 19.9 and CEA tumour markers. Samples of the tumour were taken for pathology and HER2 receptors examination. 
A

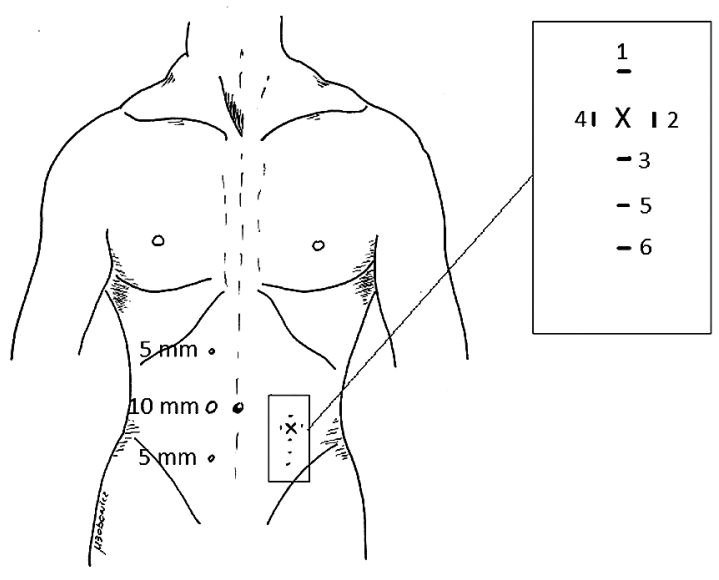

Figure 1. A - placement of trocars, B - placement of transabdominal stitches used to fix the jejunum to the abdominal wall

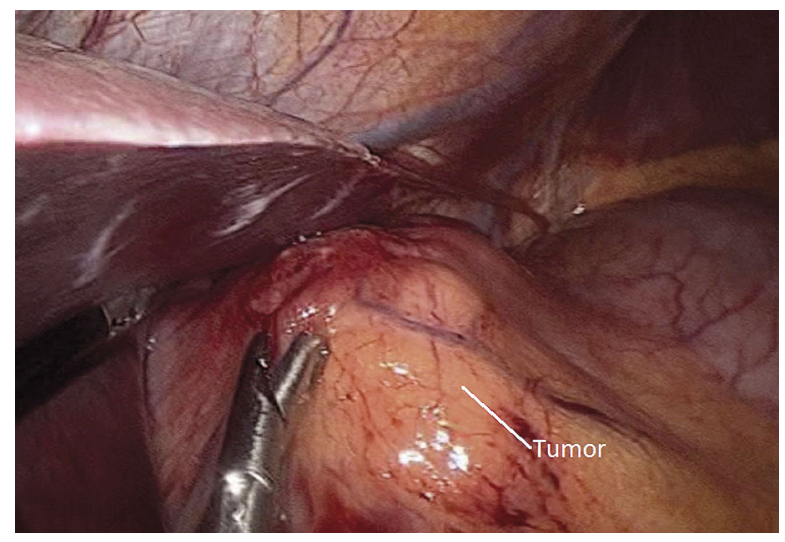

Figure 2. Inspection of the abdominal cavity

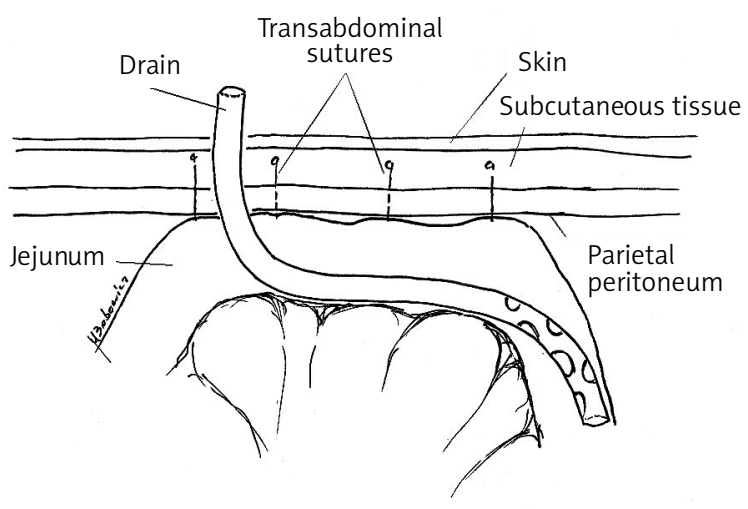

Figure 3. Transsection through the abdominal wall and jejunum showing placement of the additional 'anti-torsion' stitches

\section{Laparoscopic feeding jejunostomy - technique}

The jejunum was assessed starting from the Treitz ligament and a length of about $40 \mathrm{~cm}$ was established. The jejunum mobility and adjacency to the abdominal wall at the planned jejunostomy point was checked. Figure $1 \mathrm{~B}$ presents the diamond-like placement of transabdominal stitches used to fix the jejunum to the abdominal wall. Four stitches were applied in the consecutive marked order. First, stitches 1-3 were applied but not tied using 2.0 Novafil $^{\text {TM }}$ sutures on a straight cutting needle (Covidien). Then the Cystofix ${ }^{\circledR}$ TUR was inserted via the abdominal wall to the abdominal cavity using its introducer. Then it was introduced into the jejunal lumen with some help of two graspers. The catheter was advanced about $20 \mathrm{~cm}$ into the jejunum. The introducer was removed and the catheter was fixed to the skin with two independent 2.0 Novafil $^{\text {TM }}$ sutures. The jejunum was then pulled against the abdominal wall with the help of three transabdominal sutures. The sutures were tied. Then the fourth transabdominal stitch was placed and fixed. All transabdominal sutures were engraved into the subcutaneous tissue via small skin punctures. Two additional sutures (2.0 Novafil ${ }^{T M}$ sutures) number 5 and 6 (Figure 1 B) were placed about 4 and $8 \mathrm{~cm}$ from the jejunostomy site going through the whole thickness of the jejunal wall and the parietal peritoneum, aiming at the prevention of jejunal torsion (Figure 3). Jejunostomy patency was checked with $20 \mathrm{ml}$ of normal saline.

\section{Closure}

Drains were not placed. Standard sutures for wound closure were used.

\section{Results}

Total operating time was $45 \mathrm{~min}$. There was no blood loss. There were no intraoperative complications. The short-term outcome was good. The only adverse event was one jejunostomy wound infection that responded well to oral antibiotics. There were no mortalities. The microjejunostomy was patent during the observation period in both patients. The pathology examination in both cases showed squamous cell cancer of the oesophagus. Examination of the peritoneal fluid showed no cancer cells in both patients. Based on all the findings from the preoperative imag- 
ing, gastroscopy and staging laparoscopy, both patients were qualified for palliative treatment.

\section{Discussion}

Frequently malnutrition and cachexy are among the first symptoms of upper Gl tumours [6]. Patients with inoperable upper $\mathrm{Gl}$ cancer or on the border of operability are candidates for enteral or parenteral feeding, and when it might exceed 6 weeks enteral feeding should be favoured.

With the progress of minimally invasive techniques the idea of laparoscopic feeding jejunostomy emerged. Like every laparoscopic operation, it has its benefits and limitations. Certainly, one of its benefits is that it can be performed during the exploratory or staging laparoscopy in oncological patients with borderline operability [7].

There are multiple techniques available to perform the laparoscopic feeding jejunostomy $[4,5$, 8-18]. For example, some surgeons would prefer laparoscopy-assisted operations with micro-laparotomy and formation of the jejunostomy with the catheter's serosal tunnelization outside the abdominal cavity [8-10]. Others would use totally laparoscopic techniques $[4,5,11-18]$. It all depends on the surgeon's personal preference, and manual and technical ability.

As the described laparoscopic feeding jejunostomy was planned as part of staging laparoscopy, the authors preferred the totally laparoscopic approach. The proposed technique has most of the benefits of laparoscopic feeding jejunostomy with some steps added from the open operation making the procedure easier to perform. The authors made three major changes to the previously described techniques. Firstly, a Cystofix ${ }^{\circledR}$ TUR (B. Braun Melsungen AG) catheter was used to allow easy introduction of the tube into the peritoneal cavity and into the jejunal lumen. Secondly, the transabdominal stitches on the straight needle were used to make the technique easier. The stitches were engraved into the subcutaneous tissue, allowing use of nonabsorbable stitches. Thirdly, 'anti-torsion' stitches were applied to the caudal part of the stomy. Most importantly, the described technique avoids a minilaparotomy, and therefore the perioperative stress response and pain should be more limited, which is one of the main goals of palliative procedures. In the situation when the availability of utilities such as the Endo Stitch or
T-fasteners is limited, the use of transabdominal sutures makes the stitching much easier. Each stitch's inlet and outlet were placed in very close proximity on the skin surface to enable engraving of the suture into the subcutaneous tissue via a small skin incision. The skin punctures did not require stitching. These transabdominal sutures placed in a diamond shape provided adequate and secure adherence of the jejunum to the parietal peritoneum and allowed formation of adhesions and therefore prevented leaks from the jejunal ostium.

The choice of the tube or catheter used to create the jejunostomy is difficult and the literature does not support any of the applications in particular due to the heterogeneity of the equipment used in the available studies. The authors' choice of the $15 \mathrm{Ch}$ Cystofix ${ }^{\circledR}$ TUR (B. Braun Melsungen AG) catheter was dictated by two considerations. Firstly, it has an integral introducer and therefore it does not require any additional ports or minilaparotomies to allow insertion into the abdominal cavity. The introducer is also used to puncture the jejunum and to introduce the catheter into its lumen. It provides a good seal in the first postoperative days when it is most crucial. Secondly, the diameter of the catheter provides good patency with more thickened fluids, allowing prolonged use of the microjejunostomy.

Following laparoscopic feeding jejunostomy some authors describe jejunal volvulus as a complication requiring a re-laparoscopy or laparotomy [19-21]. To prevent jejunal torsion and volvulus, the authors placed two additional intra-abdominal stitches $4 \mathrm{~cm}$ and $8 \mathrm{~cm}$ in the caudal direction starting from the jejunostomy ostium. Our team's experience from open feeding jejunostomies shows that this modification of the technique prevents jejunal volvulus.

The meta-analysis by Han-Guerts et al. shows complication rates at the level of $17 \%$ with predominant wound infection and catheter dislodgement [22]. In the two presented patients it was a predominant complication as well and further studies on larger series of patients are required to prove its safety and benefits.

\section{Conclusions}

1. Totally laparoscopic feeding jejunostomy as a minimally invasive technique being part of staging laparoscopy is feasible with relatively short additional operating time. 
2. Use of transabdominal stitches is a crucial part of the procedure that makes the operation easier to perform.

3. Our proposal is the use of two additional 'anti-torsion sutures'.

4. Use of the Cystofix catheter allows easy introduction of the catheter into the abdominal cavity and the jejunal lumen, providing a good seal at the same time.

5. Further studies on large groups of patients are required to assess long-term outcomes of the proposed modified technique.

\section{References}

1. Moore F, Feliciano D, Andrassy R, et al. Early enteral feeding, compared with parenteral, reduces postoperative septic complications: results of a meta-analysis. Ann Surg 1992; 216: 172-80.

2. Cangelosi MJ, Auerbach HR, Cohen JT. A clinical and economic evaluation of enteral nutrition. Curr Med Res Opin 2011; 27: 413-22.

3. Mazaki T, Ebisawa K. Enteral versus parenteral nutrition after gastrointestinal surgery: a systematic review and meta-analysis of randomized controlled trials in the English literature. J Gastrointest Surg 2008; 12: 739-55.

4. Allen JW, Ali A, Wo J, et al. Totally laparoscopic feeding jejunostomy. Surg Endosc 2002; 16: 1802-5.

5. O’Regan PJ, Scarrow GD. Laparoscopic jejunostomy. Endoscopy 1990; 22: 39-40.

6. Bozzetti F; SCRINIO Working Group. Screening the nutritional status in oncology: a preliminary report on 1,000 outpatients. Support Care Cancer 2009; 17: 279-84.

7. Hori Y; SAGES Guidelines Committee. Diagnostic laparoscopy guidelines. Surg Endosc 2008; 22: 1353-83.

8. Morris JB, Mullen JL, Yu JC, et al. Laparoscopic guided jejunostomy. Surgery 1992; 112: 96-9.

9. Ellis LM, Evans DB, Martin D, et al. Laparoscopic feeding jejunostomy tube in oncology patients. Surg Oncol 1992; 1: 245-9.

10. Denzer U, Mergener K, Kanzler S, et al. Mini-laparoscopically guided percutaneous gastrostomy and jejunostomy. Gastrointest Endosc 2003; 58: 434-8.

11. Albrink MH, Foster J, Rosemurgy AS. Laparoscopic feeding Jejunostomy: also a simple technique. Surg Endosc 1992; 6: 259-60.

12. Sangster W, Swanstrom L. Laparoscopic-guided feeding jejunostomy. Surg Endosc 1993; 7: 308-10.

13. Saiz AA, Willis IH, Alvarado A, et al. Laparoscopic feeding jejunostomy: a new technique. J Laparoendosc Surg 1995; 5: 241-4.

14. Hotokezaka M, Adams RB, Miller AD, et al. Laparoscopic percutaneous jejunostomy for long term enteral access. Surg Endosc 1996; 10: 1008-11.

15. Gui GP, Cheruvu CV, Fiennes AG. Surgical technique. Intracorporeal laparoscopic-guided feeding jejunostomy. Ann R Coll Surg Engl 1997; 79: 462-3.

16. Nguyen NT, Schauer PR, Wolfe BM. Laparoscopic needle catheter jejunostomy. Br J Surg 2000; 87: 482-3.
17. Grondona P, Andreani SM, Barr N, et al. Laparoscopic feeding jejunostomy technique as part of staging laparoscopy. Surg Laparosc Endosc Percutan Tech 2005; 15: 263-6.

18. Jenkinson AD, Lim J, Agrawal N, et al. Laparoscopic feeding jejunostomy in esophagogastric cancer. Surg Endosc 2007; 21: 299-302.

19. Strickland GF, Greene FL. Needle-catheter jejunostomy for postoperative nutritional support. South Med J 1986; 79: 1389-92.

20. Sivasankar A, Johnson M, Jeswanth S, et al. Small bowel volvulus around feeding jejunostomy tube. Indian J Gastroenterol 2005; 24: 272-3.

21. Potter MB, Bowers SB, Pruitt A. Internal hernia with small bowel volvulus in a patient with altered gut motility: a complication of direct percutaneous endoscopic jejunostomy. Dig Dis Sci 2007; 52: 1910-3.

22. Han-Geurts IJM, Lim A, Stijnen T, et al. Laparoscopic feeding jejunostomy. A systematic review. Surg Endosc 2005; 19: 951-7. 